\title{
Trigonometric parallaxes in the TW Hydrae Association
}

\author{
C. Ducourant,${ }^{1}$ R. Teixeira, ${ }^{2}$ G. Chauvin, ${ }^{3}$ A. Krone-Martins, ${ }^{4}$ \\ J. F. Le Campion, ${ }^{1}$ P. Galli, ${ }^{2}$ I. Song, ${ }^{5}$ and B. Zuckerman ${ }^{6}$ \\ ${ }^{1}$ Observatoire Aquitain des Sciences de l'Univers, CNRS-UMR 5804, BP 89, 33270 Floirac, \\ France \\ email: ducourant@obs.u-bordeaux1.fr \\ ${ }^{2}$ Instituto de Astronomia, Geofísica e Ciências Atmosféricas, Universidade de São Paulo, \\ Rua do Matão, 1226 Cidade Universitária, 05508-900 São Paulo SP, Brazil \\ ${ }^{3}$ Laboratoire d'Astrophysique, Observatoire de Grenoble, BP 53, F-38041 Grenoble Cédex 9, \\ France \\ ${ }^{4}$ SIM, Facultade de Ciencias da Universidade de Lisboa, Campo Grande, Edifício C1, \\ gabinetes 1.4.21 e 1.4.39, P-1749-016 Lisbon, Portugal \\ ${ }^{5}$ The University of Georgia, Athens, GA 30602, USA \\ ${ }^{6}$ University of California, Physics and Astronomy Building, 430 Portola Plaza, Box 951547, \\ Los Angeles, CA 90095-1547, USA
}

\begin{abstract}
We have conducted a program of trigonometric distance measurements to 13 members of the TW Hydrae Association (TWA), which will enable us (through back-tracking methods) to derive a convincing estimate of the age of the association, independent of stellar evolutionary models. With age, distance, and luminosity known for an ensemble of TWA stars and brown dwarfs, models of early stellar evolution (which are still uncertain for young ages and substellar masses) will then be constrained by observations over a wide range of masses $(0.025$ to $\left.0.7 \mathrm{M}_{\odot}\right)$.
\end{abstract}

Keywords. astrometry, stars: distances, stars: kinematics, stars: low-mass, brown dwarfs, stars: evolution

\section{Introduction}

Since the discovery of the young $(\sim 10 \mathrm{Myr})$, nearby $(\sim 100 \mathrm{pc})$ association TW Hydrae (TWA) by Kastner et al. (1997), important progress has been made as regards the identification of young stars near the Sun. At present, more than 200 young, nearby stars have been catalogued. Their ages and proximity make these stars ideal to study the formation mechanisms of brown dwarfs and planets. The key parameter for all studies is the distance, which can only be directly determined through trigonometric parallax measurements. Members of young and nearby associations represent ideal targets to conduct dynamical measurements of young, low-mass binaries such as HD 98800 (late-K type), AB Dor AC, and TWA22 (Bonnefoy et al. 2009; Teixeira et al. 2009). The purpose of our work is to calibrate the predictions of evolutionary models, which are still uncertain for young ages and substellar masses. Age estimates for TWA in the literature are often based on evolutionary models and span a wide range, e.g., 5-12 Myr (Song et al. 2003) or 8-20 Myr (Boden et al. 2005). However, Ortega et al. (2002) showed that, for a sufficiently young and nearby moving group like $\beta$ Pictoris, a 'trace-back' age can be derived that is independent of evolutionary models. This property makes this method the most reliable approach for measuring ages of young stars through trace-back of its members over time until they occupy a minimum volume in space. In this paper, we 
show that the number of objects used in the trace-back method is of fundamental importance to robustly constrain the age of the association, essentially because this method is highly sensitive to peculiar velocities of objects within the cluster and to measurement errors. With 13 additional parallaxes in TWA, it will be possible to derive the age of the association based on a sample of 20 stars with known parallaxes (four based on Hipparcos measurements), 2M1207 (Ducourant et al. 2008), SSSPM1102 (Teixeira et al. 2008), TWA22 (Teixeira et al. 2009), and 13 from this work. This will ensure a very good estimate of the dynamical age of TWA, with an uncertainty of 1 Myr or better. Here we present the first step of this project: trigonometric parallax measurements of 13 TWA members.

\section{Observations}

Astrometric and photometric $(V, R, I)$ observations were performed in direct imaging mode with the ESO/NTT telescope. For the astrometric project, nine observational epochs were acquired, with a total of 36 half nights spread over almost three years between 2007 and 2010. Around 3730 exposures were taken, concentrated in 13 selected directions corresponding to the selected TWA members for which parallax measurements were required. Observational epochs were required on specific dates to maximize the parallactic factors for most targets. All observations were obtained around transit to minimize differential color refraction effects. Multiple exposures taken over three nights were obtained during each epoch to average out atmospheric effects and enhance the signal-to-noise $(\mathrm{S} / \mathrm{N})$ ratio. For most objects, two exposure times were selected, a short exposure aimed at optimizing the $\mathrm{S} / \mathrm{N}$ ratio of the bright targets, and a longer one to optimize the $\mathrm{S} / \mathrm{N}$ ratio of the faint surrounding stars.

\section{Astrometry}

CCD frames were measured using the DAOPHOT II package to fit a point-spread function, and the catalogs derived from the measurements were cross-correlated and compiled into a meta-catalog containing, for each object in the field, the object's measurements on each CCD frame. Next, all measurements were globally reduced using a block-adjustmenttype iterative procedure (Ducourant et al. 2007, 2008) to simultaneously compute the unknown stellar parameters of all stars (i.e., correction to standard coordinates, proper motions, parallaxes) and the unknown plate parameters of all frames $(2 \times 3$ or $2 \times 6$ constants per frame).

Subsequently, a statistical conversion from relative to absolute parallaxes and proper motions, based on the Besançon Galaxy model (Robin et al. 2003, 2004), was applied to derive final estimates of the absolute proper motions and trigonometric parallaxes (see, for a detailed description, Ducourant et al. 2007).

\section{Results}

We compared the preliminary distances derived in this work with the distances determined based on a convergent-point analysis by Mamajek (2005): see Fig. 1. There is general agreement and a clear coherence of the distances derived based on the convergentpoint analysis. The large discrepancy between Mamajek's distance and ours for TWA12 remains within $3 \sigma$ and probably results from a poor proper motion used in the convergentpoint analysis. 


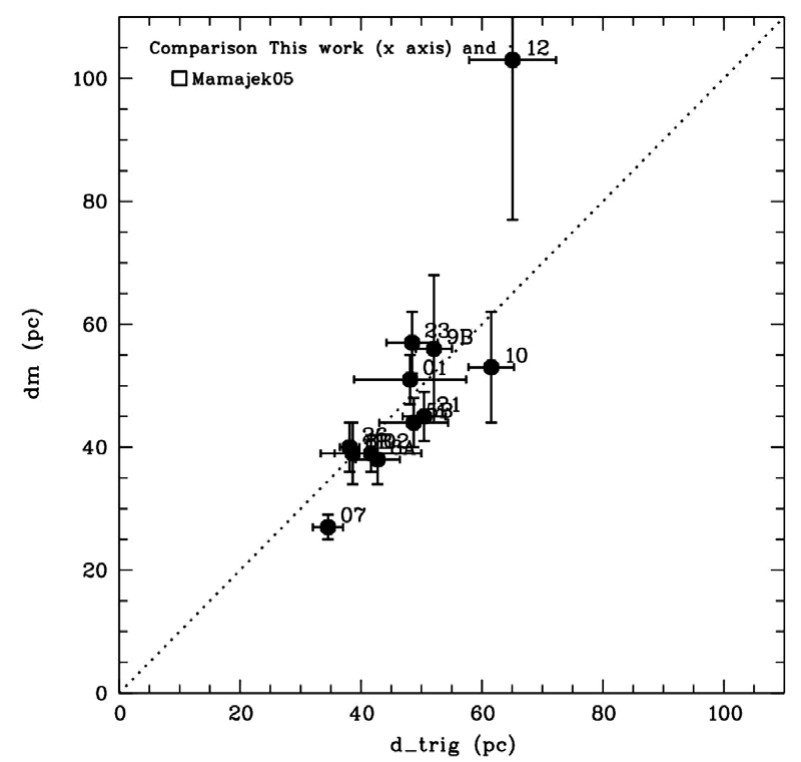

Figure 1. Comparison of trigonometric distances determined in this work for the 13 TWA targets $\left(d_{\mathrm{trig}}\right)$ with the distances determined based on a convergent-point analysis by Mamajek (2005) $(\mathrm{dm})$. The dotted line represents the diagonal where one would expect the points to be located.

The proper motions derived in this work for any star in our fields were compared with the recent release of the proper-motion catalog SPM4 (Girard et al. 2011). We present this comparison in Fig. 2. Note that the general agreement is excellent.
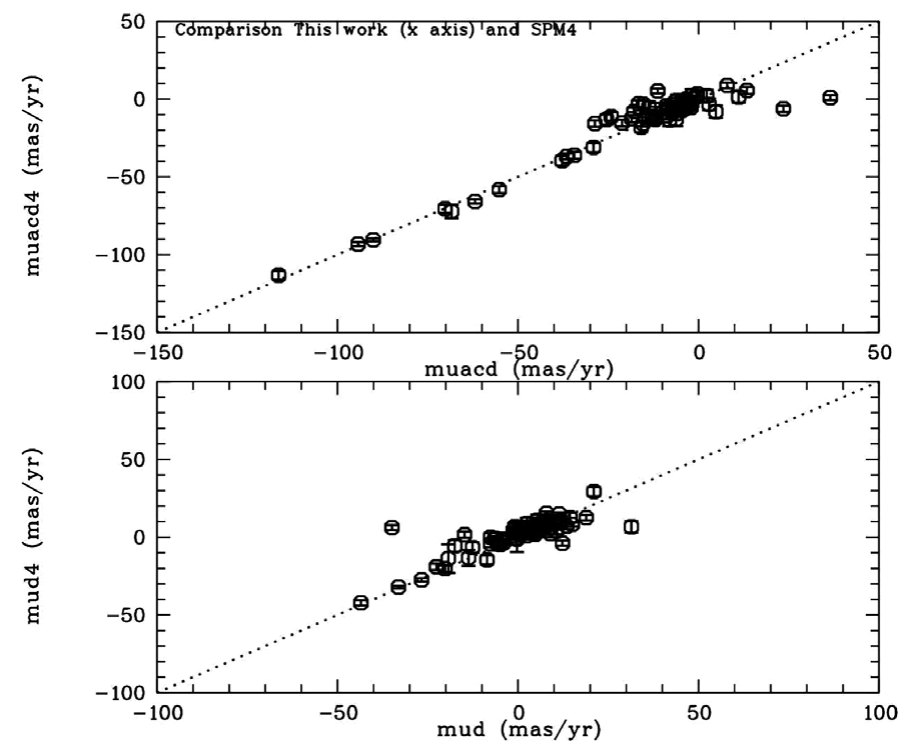

Figure 2. Comparison of proper motions $\mu_{\alpha} \cos (\delta)$ (top) and $\mu_{\delta}$ (bottom) of stars in the TWA target fields determined in this work (abscissa) with the proper motions from SPM4 (ordinate).

The first step in our project to determine the age of the TWA association has been completed and our group continues with the final step, which aims at determining the age of TWA through trace-back methods. In Fig. 3, we present the result of a simulation 
of the TWA cluster's age determination, based on 25 stars located at 60 pc from the Sun in a sphere with a radius of $14.5 \mathrm{pc}$, with common proper motions and random peculiar velocities $\left(2 \mathrm{~km} \mathrm{~s}^{-1}\right)$, evolved over 8.3 Myr. Next, an observation of the 25 members was simulated (yielding $\alpha, \delta, \mu, \pi)$ and Gaussian random errors $\left(\sigma_{\pi}=1\right.$ mas, $\sigma_{\mu}=1$ mas $\left.\mathrm{yr}^{-1}\right)$ were added to the simulated observations. Finally a trace-back method was applied to randomly selected subsamples (1000 trials) of four and 16 cluster stars to determine the age corresponding to the minimum volume occupied. In this diagram we present the resulting age histograms. The vertical line indicates the true cluster age. This result demonstrates the need to use a larger sample of stars with known accurate parallaxes than analyzed by de la Reza et al. (2006; four stars only) to properly constrain the age determination of TWA, because the trace-back method is highly sensitive to peculiar velocities of objects within the cluster and to parallax errors. It also shows that a sample containing only four stars for the age determination is not sufficiently reliable.

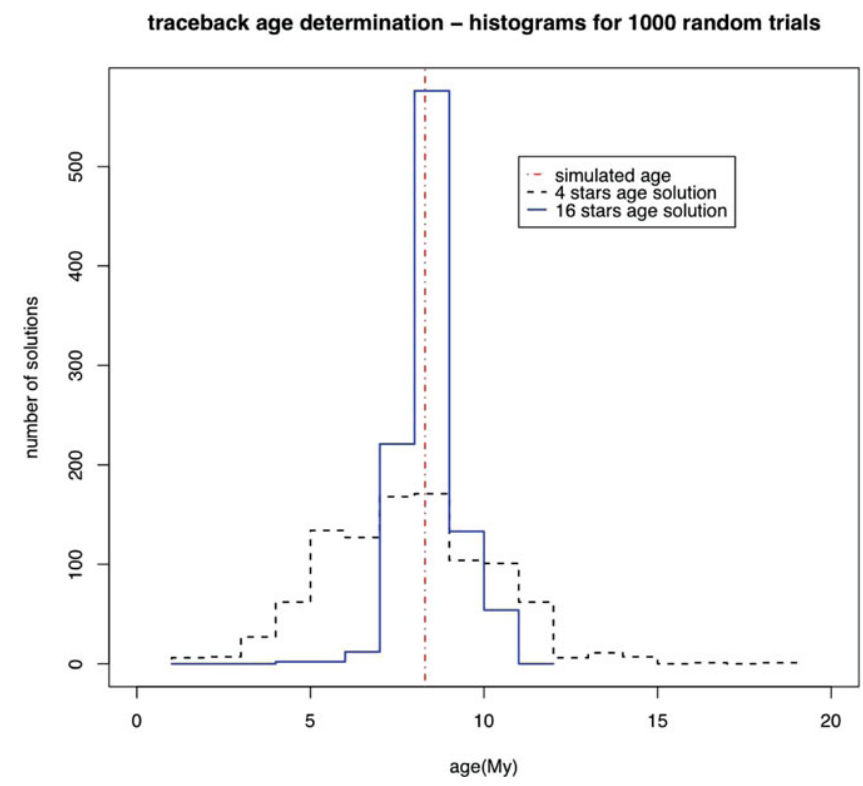

Figure 3. Trace-back age of a simulated TWA association.

\section{References}

Bonnefoy, M., Chauvin, G., Dumas, C., et al. 2009, A\&A, 506, 799

Ducourant, C., Teixeira, R., Hambly, N. C., et al. 2007, A\&SA, 470, 387

Ducourant, C., Teixeira, R., Chauvin, G., et al. 2008, A\&A, 477, L1

Girard, T. M., van Altena, W. F., Zacharias, N., et al. 2011, AJ, 142, 15

Mamajek, E. E. 2005, ApJ, 634, 1385

Ortega, V. G., de la Reza, R., Jilinski, E., \& Bazzanella, B. 2002, ApJ, 575, L75

de la Reza, R., Jilinski, E., \& Ortega, V. G. 2006, AJ, 131, 2609

Robin, A. C., Reylé, C., Derrière, S., \& Picaud, S. 2003, A\&A, 409, 523

Robin, A. C., Reylé, C., Derrière, S., \& Picaud, S. 2004, A\&A, 416, 157

Song, I., Zuckerman, B., \& Bessell, M. S. 2003, ApJ, 599, 342

Teixeira, R., Ducourant, C., Chauvin, G., Krone-Martins, A., Song, I., \& Zuckerman, B. 2008, $A \mathscr{E} A, 489,825$

Teixeira, R., Ducourant, C., Chauvin, G., Krone-Martins, A., Bonnefoy, M., \& Song, I. 2009, $A \& A, 503,281$

Zuckerman, B. \& Song, I. 2004, ARA\&A, 42, 685 